\title{
Ventroanterior Nucleus of the Thalamus
}

National Cancer Institute

\section{Source}

National Cancer Institute. Ventroanterior Nucleus of the Thalamus. NCI Thesaurus. Code C33861.

A motor nucleus that is part of the ventral tier of the lateral thalamic nuclei group, which connects with the corpus striatum. 